\title{
ProxyCare: Integrating Informal Care into Formal Settings
}

\author{
Florian Güldenpfennig, Francisco Nunes, and Geraldine Fitzpatrick \\ Vienna University of Technology \\ HCI Group, Institute for Design and Assessment of Technology \\ Vienna, Austria \\ \{florian.gueldenpfennig, francisco.nunes, geradline.fitzpatrick\}@tuwien.ac.at
}

\begin{abstract}
Chronic care is complex and involves patients, carers, and clinicians. Technology for chronic care has been developed to support formal and informal care settings. However, the integration between these two care settings has rarely been investigated. In this paper, we describe ProxyCare, an informal care technology for people living with dementia (PwD), which was successfully integrated in a formal care setting - a nursing home - for four weeks. Reflecting on our design considerations and pilot, we draw implications for the design of informal care technologies that integrate well into institutionalized settings.
\end{abstract}

Keywords-informal care; formal care; chronic care; dementia; monitoring system.

\section{INTRODUCTION}

Living with a chronic condition requires great effort. Care does not end after a consultation, but rather continues in everyday life, through the work of patients and carers, in building a life with quality [1]. Patients and carers are likely to take an active role in health management: monitoring changes in the ill body, acting on symptoms, managing treatment, and dealing with the psychological, physical, and practical consequences of living with a chronic condition [2]. Informal care $^{1}$, performed by patients and carers (i.e., family and close friends), and formal care, performed by professionals in clinics, hospitals or nursing homes, are closely intertwined. Professionals are likely to provide directions for informal care, and at the same time try to understand the perspectives of patients and carers, to provide good recommendations [1].

Technologies for informal care have been able to help patients and carers to live with their chronic conditions. The blood glucose meter is a good example of an informal care technology that helps patients, and sometimes carers, monitor blood glucose and so control diabetes. Informal care technologies have been used, for example, to: learn about the condition from educators [3]; adjust care based on symptom monitoring [4]; or detect emergency disease states [5]. Most of these technologies, have been designed for patients to self-care

\footnotetext{
${ }^{1}$ Informal care in this paper refers to the care performed by patients or carers living with chronic conditions. This care appears as a complement to formal care that is performed by professionals, and focuses on achieving a good quality of life despite health issues. In the case of ProxyCare, informal care is performed by the patient's relatives and not by the patient herself (self-care).
}

[3]-[5], but there are also technologies designed for carers, to be able to better look after patients [6]-[8].

The studies around informal care technologies are usually located around the home, or in everyday life, supporting patients and carers in the different situations of their life. It is not common though to find studies about informal care in formal settings. It is as if, when people moved to institutional settings such as a nursing home, they abandoned their informal care practices and devices [9]. What happens in reality is that some informal care practices are transformed, while others continue to exist [9]. Ignoring informal care practices in formal settings may decrease the quality of care, so it is relevant to study how the two settings can be integrated. This paper explores this research space by creating an informal care technology to be used in a formal setting.

The contribution of this paper is twofold. First of all, it contributes with a fully implemented prototype named ProxyCare (see Fig. 1-3) that integrated a self-care technology into a formal setting. Drawing on a case study of this technology in use, we then derive implications regarding the design of systems that aim to integrate institutionalized with informal care.

This paper is organized in 6 sections. The next section details our study scenario (Section II). Section III describes the ProxyCare prototype. Section IV presents the findings of our pilot. Section V discusses our results and the implications for design. And finally, section VI concludes the paper.

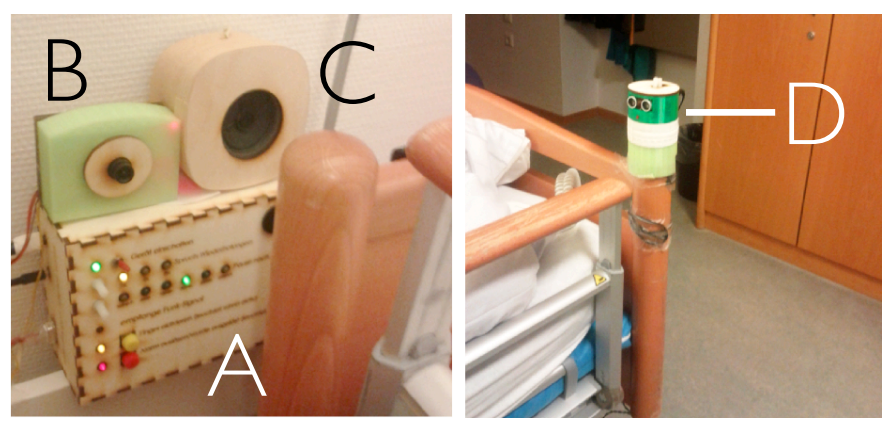

Fig. 1. The ProxyCare monitoring system for people with dementia (PwD) installed at a nursing home bed. Left: ProxyCare control module (A), logging device (B), and audio module (C). Right: One of the system's wireless sensor sattelites (D) for detecting motion. See Section III for a detailed description. 


\section{STUDY SCENARIO}

\section{A. Introduction to Care of Dementia (in General)}

Dementia is a complex degenerative condition that affects the brain. Apart from its medical characteristics, affecting memory and cognition, the condition impacts the life of patients and carers in a number of different ways [6][7]. As the condition progresses, people are likely to lose important abilities, resulting in a dependency that progressively grows.

Part of dementia care is clinical. It is about understanding the evolution of the condition, and adapting treatment to the progression. Another part is informal care - provided by family and friends, as patients lose the ability to care for themselves making lifestyle adaptations, managing emotions, and building an adequate life, despite the health constraints.

\section{B. Our Specific Case}

This paper is based on the case of a lady we name Paula. Paula is 92 years old and suffers from multiple severe conditions including advanced dementia. She has been living in a nursing home for the past three years. The acute fracture of her hipbone led to a particular precarious situation: Paula will damage her hipbone reconstruction if she puts weight on her legs, but she cannot remember this due to her dementia. She keeps trying to get out of bed, and to go to the toilet in the nursing home (even though she has a urinal catheter). Putting weight on her legs highly increases the chance of a repeated fracture and inner bleeding as her injury is very complicated. To address this issue, Paula's bed was augmented with standard bed-rail barriers on both sides. However, this resulted in even more risk as she kept on attempting to climb over this barrier. On several instances, she fell to the ground and had to be rescued by the medical staff.

There were not enough caregivers (nursing home staff and informal carers) available to look after Paula constantly (day and night), and the nursing home did not have any monitoring devices other than a floor mat with an integrated weight sensor. This technology could have helped, but the alarm of the mat would only go off after Paula stepped on it, and so damage would have already been inflicted to her hipbone. The possibility of securing Paula to the bed was considered, but this option could not be taken into practice due to legal reasons.

To address these concerns, the family requested that we build an informal care technology tailored towards Paula's specific situation (considering her medical condition and the need to build a technology to be integrated in an institution). We argue this is an informal care technology, because it was initiated by non-professionals, who wanted it as a proxy to support and extend their care role when they could not be there. There is a clinical/cognitive issue underlying this problem, that of not being able to remember, but the answer required is in changing everyday life behaviors, and so part of informal care. This system was built with the support of the ward physician and other staff, including the head of the institution.

We go on to describe the result of our engineering effort, which was implemented following a user-centered design process.

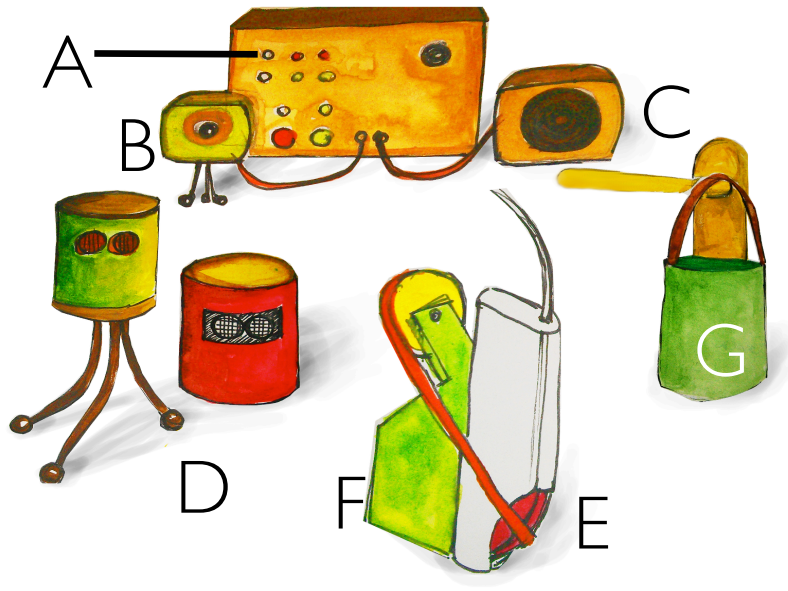

Fig. 2. Schematic overview of the ProxyCare system comprised of components A-G.

\section{PROXYCARE SYSTEM}

ProxyCare is a modular informal care technology for monitoring the behavior of PwD. The system notices when the patient attempts to stand by detecting when she starts to move her legs out of the bed, and plays a voice message, recorded by a family carer, persuading her not to get up. In contrast to related systems [10], the technology was operated by informal carers and is aimed to protect the patient from injuries, caused by standing after a hip reconstruction surgery (cf. Section II.B).

It was particularly important for this system not to hinder the professional staff during their daily care routine. On the contrary, ProxyCare is intended to enable a certain form of collaboration between informal and professional carers to increase the quality of life of PwD.

These features and requirements are reflected in the design of ProxyCare's different components as depicted in Fig.1-3 and as outlined in the following:

- A: The main or control module collects and coordinates all signals that are generated within ProxyCare. These signals comprise incoming alarms from satellite modules $(D, G)$ and directly entered user input via the main module's interface: alarm the system, pause the ProxyCare system, set maximum numbers of alarms, set idle time between two alarms, set the number of repetitions of the audio message (see below). In addition, the main module sends triggers to the alarm actuator $(\mathrm{F})$, logging module $(\mathrm{B})$, and the audio module (C).

- $\quad$ B: The logging module can optionally be plugged into the control module $\mathrm{A}$ and records events that were triggered either by the wireless ${ }^{2}$ satellite modules (see $\mathrm{D}$ and $\mathrm{G}$ ) or by the user interface of the control module A. It features an optional camera for capturing photos of the scene when an alarm was triggered. This function was integrated for debugging purposes and to ensure that the patient is not harassed by unnecessary

\footnotetext{
${ }^{2}$ The communication between ProxyCare modules A, D and G is done wirelessly using XBee transceivers.
} 
false alarms. An attachable/optional tripod supports orientating the camera lens.

- $\quad \mathrm{C}$ : The audio module can optionally be plugged into control module A and plays personalized audio messages to the patient when the alarm is triggered (i.e., when the patient attempts to get up). In Paula's case the chosen message was "mother, please remember that you are connected to a urinal catheter, stay in bed and wait for the nurse", with the voice of the patient's daughter.

- D: Sensor modules (movement detected by ultrasonic range sensors). These are wireless 'satellites' that automatically connect to control module (A) when supplied with power (battery pack or outlet).

- E: Conventional emergency button. This button is owned by the institution and connected to the ward alarm system. It is designed for patients, but Paula did not use it due to her advanced dementia.

- F: Alarm Actuator ('mechanical finger'), triggers emergency button (E) by applying traction. Hence, the motor of this module causes the same effect as if the button was pressed by a human finger.

- G: Pause Bag to be hung on the door handle and connected wirelessly with main module $A$. The opening of the door and thus door movement is registered by a built-in accelerometer. Pause Bag then pauses ProxyCare for 5 (or more) minutes to avoid false alerts to be triggered by visitors entering the room. This mechanism was implemented in particular so as not to interfere with the staff's daily routine and hence increase their acceptance of ProxyCare.

\section{PROXYCARE STUDY}

\section{A. Setup}

ProxyCare was piloted for four weeks (29 days) in a nursing home. The system was operated by Paula's informal carers, namely her daughter and granddaughter. During the pilot, activities were logged to ensure ProxyCare worked properly and did not harass the patient with false alarms. After four weeks, the $\log$ files were evaluated quantitatively. Interviews and informal conversations were conducted with Paula's family, the ward doctor, and a number of nurses, on a regular basis during the design and piloting of the system. Notes were taken during these events.

The institution's staff (including direction, ward doctor, and nurses) was informed about the purpose of ProxyCare and approved its pilot use in the institution. Ethical approval was not required by the institution nor our university, but we followed the ethical principles and recommendations common in healthcare studies, including the Declaration of Helsinki.
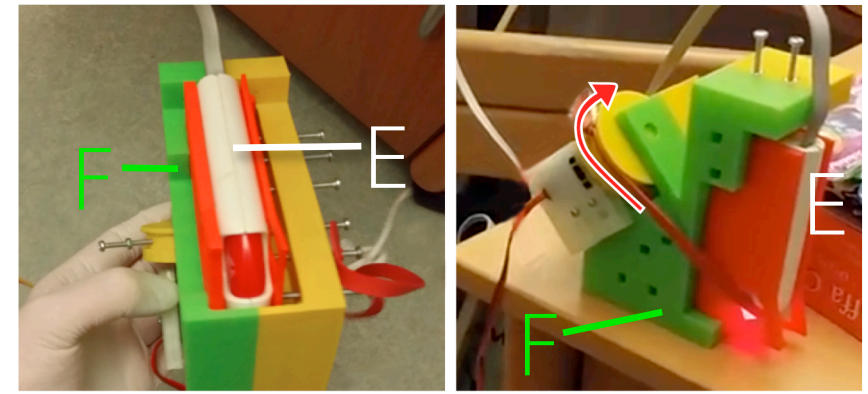

Fig. 3. ProxyCare alarm actuator (F). Emergency button (E; owned and provided by the institution) is triggered by ProxyCare's alarm actuator (F) by applying traction (Right: alarm went off as evident from the illuminated red LED).

\section{B. Findings}

Both carers - Paula's daughter and granddaughter - were satisfied with ProxyCare ${ }^{3}$. According to their account, it reassured them and alleviated some of their concerns, as they could trust the system to reliably alarm the medical staff whenever Paula attempted to climb over the bedrails. Paula's daughter tried different voice messages and reported that these messages seemed to calm her mother down and persuade her to stay in bed and wait for the nurses.

The medical staff also valued the system. The ward doctor, who had been ProxyCare's advocate from the beginning, said that he was relieved a solution could be found for the patient. All staff except one nurse (4 out of 5) reported that ProxyCare was unobtrusive and did not add extra work for them. Instead, they appreciated that the system prevented the critical incident of Paula falling on the floor and thus leading to dangerous situations, stress and finally to more work.

The unobtrusiveness of the system, facilitated by the Pause Bag (Fig. $2 \mathrm{G}$ ) hanging on the door, was also valued. The nurses were satisfied that the system would pause itself without requiring them to take any action. For them, it was practical.

In total, 72 attempts of getting out of bed were captured by the system avoiding a potential fall. No false alarms were identified, either from the logs or from observation/reports (by formal and informal carers). The system was paused, automatically by the Pause Bag 383 times; this number is a sum of all visits, including for giving meals, washing, etc. On 66 occasions ProxyCare was paused manually using a button on the main module.

The director was concerned, in the beginning, that the system would damage, or require them to change, their infrastructure. As the prototype was available, they were happy to recognize that no invasive modifications were needed. Instead, ProxyCare gave alarms by mechanically triggering the emergency button of the nursing home, in the same way a person would. Furthermore, drawing on and integrating already existing technologies (i.e., the emergency button), saved costs and, most importantly, made triggering alarms most reliable.

\footnotetext{
${ }^{3}$ Due to Paula's dementia it was not possible to ask her directly about her acceptance of the system. However, her relatives and the ward doctor didn't observe any negative reactions to ProxyCare. On the contrary, the audio module of the system appeared to have a calming effect.
} 
After four weeks ProxyCare was removed from the nursing home as Paula partially recovered from her fracture, and the injury risk was reduced. The institution removed the technology as quickly as possible, although it could have been used for some more weeks out of precaution. The reason for suspending the use so quickly was due to a complaint of a nurse $^{4}$ who suggested, contrary to all other people involved, that the technology was making the patient feel more confused. The administration did not want to take risks and opted for its removal, once the patient stopped requiring it.

\section{DISCUSSION}

ProxyCare integrated successfully into a formal setting and was effective for its purpose. We can observe this in the number of times it stopped Paula from getting out of the bed, and from the positive opinions expressed by almost all of the people involved in this study.

We suggest that part of the success of ProxyCare is due to the way it integrated with the practices of the institution's staff. From the connection with the alarm button, to the pause starting when someone entered the room, there was great consideration during the design to ensure professional carers would accept the system. With ProxyCare, they were not interrupted, and were even able to do more with their time.

From the perspective of the informal carers, the system was also successful. It enabled them to still participate in the care of Paula although she was in a nursing home. They also felt that the provided care was not 'cold' as a 'standard medical device', but personalized to the needs of Paula. Using their own voice was especially important for calming Paula down. The system represented a care proxy for them in the institution. They felt Paula was protected, even when they were not in the institution. Hence, ProxyCare supported their concerns and needs as informal carers, which are at times neglected in institutional settings.

\section{A. Implications for the Design of Informal Care Technologies that Integrate Well in Formal Settings}

We detail here a set of design implications drawn from our observations and findings:

- Allow carers to customize the system to the patient. The carer will be aware of the personality traits of the patient, ensuring the system works adequately for him or her (all illustrated by, e.g., audio module in Fig. $2 \mathrm{C}$ or the variety of different settings for main module A).

- Make the system easy to adapt and configure by informal carers, as they will be the ones shaping the system to changes in the infrastructure of the setting (building on also previous implication item).

- $\quad$ Provide feedback to (informal) carers that the system will work as they expect. Carers will eventually go home, and the system will be working closely with the staff, so carers need to know they will not cause problems to the staff.

\footnotetext{
${ }^{4}$ According to Paula's relatives this particular nurse had a certain 'reputation' at the nursery home for enjoying conflicts and taking opposing positions.
}

- Explain the benefits of the system to the staff, considering the patient's needs and the staff's work.

- Integrate with the everyday practices of the institution's staff, introducing the least work possible, and without requiring them to learn another machine.

- Make the system modular enough that it continues to work despite changes in the structure and routines of the institution, e.g., changing beds, cleaning, etc.

- Avoid changing the existing technical infrastructure. Institutions do not wish to be held liable for bad care provision, and keeping the technical infrastructure 'untouched' is one way to assure the management that things will go on as usual.

\section{CONCLUSION}

In this paper we introduced ProxyCare, an informal care technology that was successfully integrated in an institutional setting for four weeks. We have documented the requirements we considered, how these materialized in the prototype, and discussed possible reasons for the success of the technology. Our work should be useful when developing informal care technologies for formal settings.

As the next step, we plan to further investigate our design implications incorporating multiple participants.

\section{ACKNOWLEDGMENTS}

We thank Dorothee Köstlin for her design input and assistance in conducting the study.

\section{REFERENCES}

[1] A. Mol, The logic of care: Health and the problem of patient choice. Routledge, 2008.

[2] J. Barlow, C. Wright, J. Sheasby, A. Turner, and J. Hainsworth, "Selfmanagement approaches for people with chronic conditions: a review", Patient Educ. Couns., vol. 48, no. 2, pp. 177 - 187, 2002.

[3] L. Mamykina, E. Mynatt, P. Davidson, and D. Greenblatt, "MAHI: Investigation of Social Scaffolding for Reflective Thinking in Diabetes Management," in Proc. CHI '08, Florence, Italy, 2008, pp. 477-486.

[4] R. Aarhus, S. A. Ballegaard, and T. R. Hansen, "The eDiary: Bridging home and hospital through healthcare technology," in ECSCW 2009, Springer London, 2009, pp. 63-83.

[5] J. E. Bardram, M. Frost, K. Szántó, M. Faurholt-Jepsen, M. Vinberg, and L. V. Kessing, "Designing Mobile Health Technology for Bipolar Disorder: A Field Trial of the Monarca System," in Pro CHI 2013 , Paris, France, 2013, pp. 2627-2636.

[6] P. Jordan, P. A. Silva, F. Nunes, and R. Oliveira, "mobileWAY - A System to Reduce the Feeling of Temporary Lonesomeness of Persons with Dementia and to Foster Inter-caregiver Collaboration," in HICSS 2013, pp. 2474-2483.

[7] J. Wallace, P. C. Wright, J. McCarthy, D. P. Green, J. Thomas, and P. Olivier, "A Design-led Inquiry into Personhood in Dementia," in $\mathrm{CHI}$ '13 Extended Abstracts on Human Factors in Computing Systems, New York, NY, USA, 2013, pp. 2883-2884.

[8] C.-C. Lin, M.-J. Chiu, C.-C. Hsiao, R.-G. Lee, and Y.-S. Tsai, "Wireless Health Care Service System for Elderly With Dementia," IEEE Trans. Inf. Technol. Biomed., vol. 10, no. 4, pp. 696-704, Oct. 2006.

[9] B. J. Bowers, "Family Perceptions of Care in a Nursing Home," The Gerontologist, vol. 28, no. 3, pp. 361-368, Jun. 1988.

[10] S. Nava-Muñoz, A.L. Morán, "CANoE: A Context-Aware Notification Model to Support the Care of Older Adults in a Nursing Home". Sensors, vol. 12, no. 9, 11477-11504, 2012. 